\title{
Prospects for In Situ TEM on Electrocatalyst Materials for Sustainable Energy Technologies
}

Robert Sinclair $^{1}$, Yunzhi Liu ${ }^{2}$ and Thomas F. Jaramillo ${ }^{3}$

${ }^{1}$ Department of Materials Science and Engineering, Stanford University, Stanford, United States, ${ }^{2}$ Department of Materials Science and Engineering, Stanford University, Stanford, California, United States, ${ }^{3}$ Department of Chemical Engineering, Stanford University, Stanford, California, United States

One approach to address energy-related issues is through the catalytic improvement of electrochemical conversions employed in technologies for the production or consumption of fuels, such as the hydrogen or oxygen evolution reactions (HER, OER), oxygen reduction reaction (ORR) etc. It is also important to find less expensive alternatives to highly efficient platinum catalysts. With this in mind the current research has focused on exploring the usefulness of various inorganic compounds such as metal sulphides, oxides or nitrides which are predicted by DFT calculations to be possible candidates $[1,2]$.

For reproducible electrochemical characterization, thin films of the materials of interest are deposited onto conductive substrates (e.g. [3]) and subsequently characterized by transmission electron microscopy (TEM) methods often in cross-section. The long-term goal of the latter is to provide atomic level information about the catalyzing reactions, for which in situ TEM will eventually be invaluable.

The usefulness of in situ environmental TEM is demonstrated by the structural and bonding changes observed in amorphous molybdenum sulphide thin films under exposure to hydrogen [4], as revealed by high resolution imaging and electron energy loss spectroscopy, which complement those obtained during HER testing. However, the in situ approach of electrochemical testing in a liquid environment often under harsh acidic or alkaline conditions, is much more problematic. We have shown for instance the considerable loss of catalytic material during testing of strontium iridate for OER in sulfuric acid solution [5] and of molybdenum nitride or oxynitride for ORR in perchloric acid solution [6, 7, 8]. Neither of these undergo structural or phase changes under testing, but there are significant alternations to their surface structure [5, 6]. Another interesting material is iridium oxide $\left(\mathrm{IrO}_{2}\right)$ which can be partially stabilized in the Columbite phase $\left(\alpha-\mathrm{IrO}_{2}\right)$ coexisting with Rutile phase $\left(\mathrm{R}-\mathrm{IrO}_{2}\right)$ by Pulse Laser Deposition onto a YSZ substrate, as revealed by high resolution imaging and FFT analysis [9] (e.g., Fig. 1).

In order to attempt to capture key stages of these reactions, in situ liquid cell experiments are being developed which are clearly problematic because of the harsh environments under applied voltage conditions. If successful, new information at the atomic level is certain to be generated, but the advances required to achieve reproducible data representative of the true reaction will be difficult [10].

\section{References}

[1] Abroshan, Hadi, et al. J. Phys. Chem. C. 122.9 (2018), p. 4783-4791

[2] McCrory, Charles CL, et al. J. Am. Chem. Soc 135.45 (2013), p. 16977-16987.

[3] Seitz, Linsey C., et al. Science 353.6303 (2016), p. 1011-1014.

4] Lee, Sang C., et al. ACS Nano 10.1 (2015): pp. 624-632.

[5] Liu, Yunzhi, et al. Microsc. Microanal. 26.S2 (2020) pp. 3168-3169.

[6] Liu, Yunzhi, et al. Microsc. Microanal. 25.S2 (2019) pp. 2072-2073.

[7] Kreider, Melissa E., et al. Chem. Mater. 32.7 (2020) p. 2946-2960. 


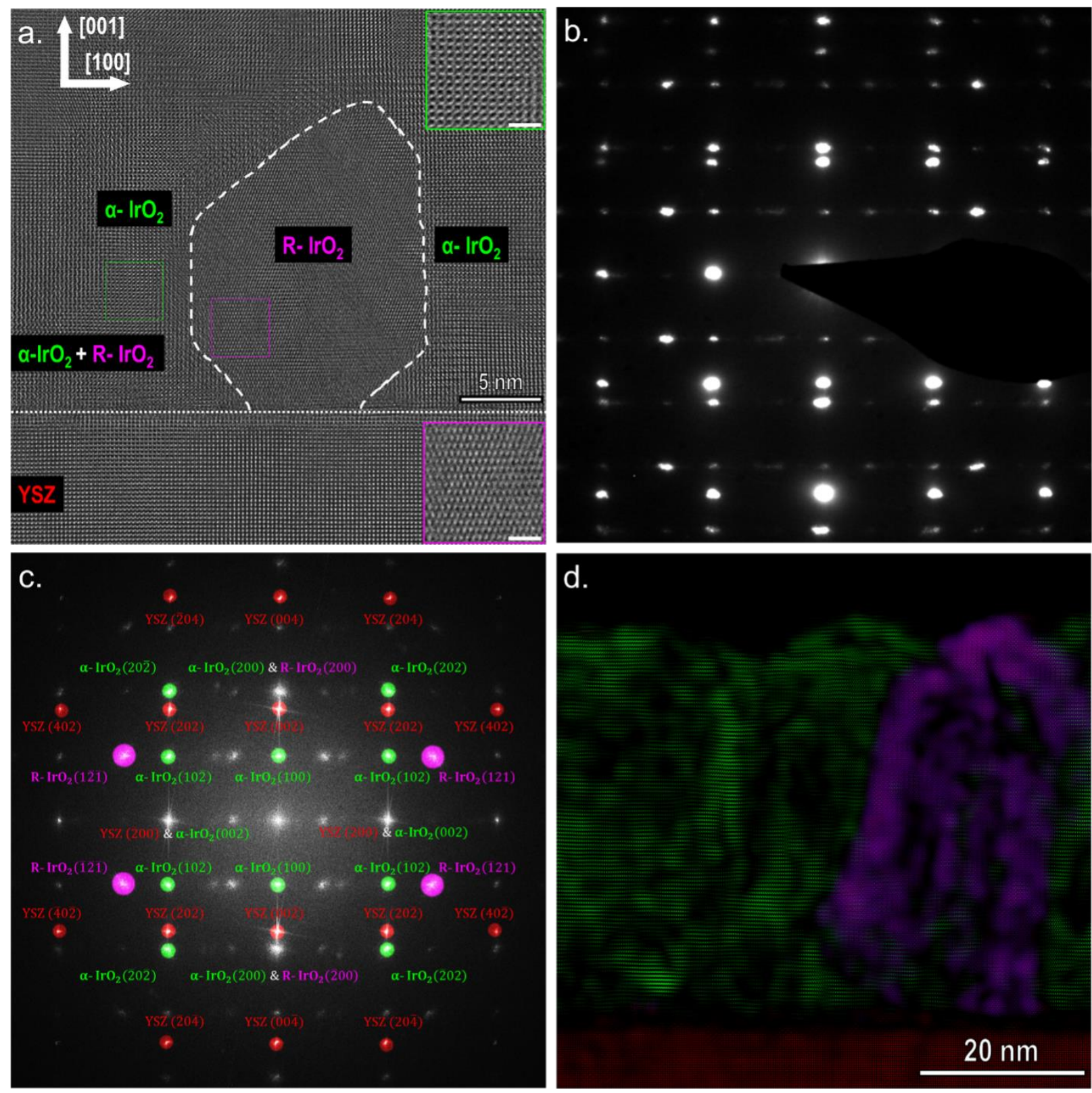

Figure 1. Aberration corrected HR-TEM images of $\mathrm{IrO}_{2}$ thin film. (a) HR-TEM image of the $\mathrm{IrO}_{2}$ thin film and YSZ substrate, with inset HR-TEM showing crystal structures of $\alpha$ - $\mathrm{IrO}_{2}$ (green) and $\mathrm{R}-\mathrm{IrO}_{2}$ (purple) accordingly. (b) Selected area diffraction pattern taken on the thin film and substrate, indexing is consistent with the FFT in (c), (c) FFT of (a) and color index of $\alpha-\mathrm{IrO}_{2}$ (green), $\mathrm{R}-\mathrm{IrO}_{2}$ (purple) and YSZ (red) accordingly. (d) FFT filtered inverse image of another region with larger field of view, showing various phases in the image. The color code and spots used for imaging are marked in (c).

[8] Stevens, Michaela B., et al. ACS Appl. Energy Mater. 3.12 (2020) p. 12433-12446.

[9] Lee, Kyuho, et al. ACS Appl. Energy Mater. (submitted)

[10] Financial support from the Toyota Research Institute - Accelerated Materials Design and Discovery (TRI-AMDD) program (Stanford University) is much appreciated. 